\title{
A Regularized Lagrangian Finite Point Method for the Simulation of Incompressible Viscous Flows
}

\author{
Jiannong Fang*, Aurèle Parriaux \\ Ecole Polytechnique Fédérale de Lausanne (EPFL), Engineering and \\ Environmental Geology Laboratory, ENAC-ICARE-GEOLEP, Station 18, \\ CH-1015 Lausanne, Switzerland
}

\begin{abstract}
In this paper we present a regularized Lagrangian finite point method (RLFPM) for the numerical simulation of incompressible viscous flows. A Lagrangian finite point scheme is applied to the projection method for the incompressible Navier-Stokes equations. The approximation of spatial derivatives is obtained by the weighted least squares method. The pressure Poisson equation with Neumann boundary condition is solved by a stabilized finite point method. A key aspect of the present approach is the periodic redistribution of the particle locations, which are being distorted by the flow. Again, weighted least squares approximation is implemented to interpolate the properties of the old particles onto the new particle locations. With the proposed regularization technique, problems associated with the flow-induced irregularity of particle distribution in the Lagrangian finite point scheme are circumvented. Three numerical examples, Taylor-Green flow, lid-driven flow in a cavity and flow through a periodic lattice of cylinders, are presented to validate the proposed methodology. The problem of extra diffusion caused by regularization is discussed. The results demonstrate that RLFPM is able to perform accurate and stable simulations of incompressible viscous flows.
\end{abstract}

Key words: Meshless/meshfree method, Finite point method, Generalized finite difference method, Incompressible viscous flows, Navier-Stokes equations, Projection method, Weighted least squares approximation 1991 MSC: 65M06, 76D05

* Corresponding author. Tel.: ++41 21693 2357. Fax: ++41 216936330. Email address: jiannong.fang@epfl.ch (Jiannong Fang). 


\section{Introduction}

Recently, in the general area of computational mechanics there is a growing interest in developing so-called meshless methods or particle methods as alternatives to traditional grid-based methods such as finite difference methods and finite element methods. The key idea of these methods is to provide numerical solutions on a set of arbitrarily distributed points without using any mesh to connect them. Mesh generation can be very costly and difficult, especially for the simulation of 3D problems with large deformation and within complex geometry. Compared to that, it is relatively simple to establish a point distribution and adapt it locally. When worked in a Lagrangian form, points used in meshless methods are often called particles because they not only function as interpolation points but also move like mass points. Depending on the methodology used to obtain discretized equations, meshless methods can be classified into two major categories: meshless strong-form methods and meshless weak-form methods. Most of meshless weak-form methods are "meshless" only in terms of the numerical approximation of field variables and they have to use a background mesh to do numerical integration of a weak form over the problem domain, which is computationally expensive. Meshless strong-form methods often use the point collocation method to satisfy the governing differential equations and boundary conditions. They are simple to implement and computationally efficient. Since they do not need any background mesh, they are truly meshless methods. However, they are often less stable and less accurate.

Among the various truly meshless methods, Smoothed Particle Hydrodynamics $(\mathrm{SPH})$ is the longest established grid free Lagrangian method. SPH was originally developed for astrophysical applications [1,2]. Since its invention, it has been extensively studied, extended and applied in many areas such as the dynamic response of elasto-plastic materials [3-5], free surface flows [6], viscous flows [7-10], solid friction [11], heat transfer [12], multi-phase flows $[13,14]$, geophysical flows [15-17], turbulence modeling [18], and viscoelastic flows $[19,20]$. For a long time, SPH was suffering from several problems: inconsistency; tensile instability; and difficulty in the treatment of boundary conditions. The latter two problems are somehow related to the first one. Various methods have been developed to improve SPH. The symmetrization and antisymmetrization in some SPH formulations [21] were first attempted to improve the accuracy of the SPH particle approximation. A reproducing kernel particle method was developed by Liu et al. [22] to reproduce consistency conditions by correcting the smoothing function which is a key component in particle approximation. Johnson and Beissel [23] proposed a normalized smoothing function algorithm. Dilts [24,25] introduced the moving least square approximation into SPH computations. Using Taylor series expansion, Chen and Beraun [26] developed a corrected smoothed particle hydrodynamics method for nonlinear 
dynamic problems. Their work was followed recently by Liu et al. [27] who proposed the so-called finite particle method (FPM) and applied it to viscous fluid flows. Zhang and Batra [28] applied a similar idea to transient problems in elastic dynamics and heat conduction. Most of the previous contributions attempting to improve $\mathrm{SPH}$ have focused on replacing directly the existing SPH particle approximations to functions and their derivatives by corrected approximations with polynomial consistency enforced. As a consequence, angular momentum and/or linear momentum are usually not strictly conserved by the discrete equations and the long-time simulations are potentially unstable. Differently, Bonet and Lok [29] presented a corrected SPH formulation using a variational framework with the advantage of preserving both linear and angular momentum. Similarly, using an energy-based approach, Fang et al. [30] derived a general set of discrete hydrodynamics equations with conservation properties to which any corrected (high-order) or coupled particle approximation scheme can be applied. From either a variational or an energybased approach, a close link is found between discrete velocity derivatives and momentum equation, which can be named as the scheme coherence principle. The scheme coherence principle ensures the conservation of momentum. However, it can not be satisfied together with the consistency requirement for the discrete momentum equation. Recently, Oger et al. [31] has shown that the scheme coherence principle could be flouted, allowing the introduction of a more accurate SPH formulation.

Finite point method (FPM) is another truly meshless method proposed by Oñate et al. [32]. FPM uses a weighted least square interpolation scheme within each point cloud, which can be easily constructed to have consistency of a desired order, and adopts the point collocation method to obtain the discrete equations. Therefore, it is easy for numerical implementation and boundary conditions can be implemented in a natural way by just prescribing boundary conditions on points placed on boundaries. Moreover, not like in the classical SPH method, particles in the finite point method do not have a mass associated and they are merely interpolation points for the field information, including density. This makes the method more flexible in terms of an easier particle management and an easier treatment of boundaries. The price to pay for these advantages is that the method is not strictly conservative anymore, as opposed to SPH. The efficiency of FPM for solving a range of problems has been reported in [33-37]. In the same spirit of the finite point method, but using a Lagrangian description, Tiwari and Kuhnert have developed the Lagrangian finite point method (LFPM) and used it in many applications such as two-phase flows [38] and fluid-structure interactions [39]. Be a strong-form method, stabilization of FPM is often necessary. An upwinding approach was used as stabilization procedure in [32]. A residual stabilization technique [33] was later proposed as an alternative stabilization procedure appropriate for FPM. The technique was further developed into the so-called finite increment calculus (FIC) approach by Oñate [40]. Application of the FIC approach to the 
solution of fluid flow problems using FPM can be found in [34,35]. Another simple modification for stabilization of the Neumann boundary conditions using equilibrium residuals at the boundaries was presented by Boroomand et al. [41].

Generally speaking, Lagrangian particle methods can handle convection dominated flows and large deformation problems very well due to their Lagrangian and adaptive nature. However, it is also known that, with a fixed support domain (or an Eulerian kernel) for each particle, it is difficult to maintain accurate interpolation based on the Lagrangian particle set, which is usually distorted by the flow map and hence loses its regularity. Sometimes particles undergo so massive amounts of strain that they do not overlap anymore in some directions. SPH solutions obtained from increasingly more irregular particle distributions will exhibit an increasing amount of numerical errors. This is the reason for the accuracy degradation of some SPH simulations. For LFPM, it is even possible to encounter the problem of ill conditioned matrix. To deal with the problem, two kinds of technique have been proposed. The main idea of the first kind is to take advantage of the adaptive distribution of particles. This kind of method includes the adaptive SPH $[42,43]$, the total Lagrangian SPH $[44,45]$, and the anisotropic neighbor searching algorithm [46]. The adaptive SPH introduces anisotropic kernel with tensor smoothinglengthes evolving automatically to follow the mean particle spacing as it varies in time, space, and direction around each particle. The efficiency of the method has not been extensively tested for a variety of flows. In the total Lagrangian SPH the neighborhood of each particle is determined from a reference configuration and remains fixed throughout the simulation. Nevertheless, in case of extremely large distortion, updating the reference configuration is necessary and may induce additional problems. The anisotropic neighbor searching algorithm aimed to select a minimal and robust set considering large anisotropy in the particle arrangement. Special treatment is needed when applying the method to find neighbors of a boundary point. The key idea of the second kind method is to restore regularity of the particle set. The regularized SPH [47] and the similar one called re-meshed SPH [48] belong to this category. The regularized SPH allows the particle distribution to be entirely redefined through a regularization process based on an optimized smoothing-length profile. In the re-meshed SPH (RSPH), the particle locations are periodically re-initialized onto a uniform grid. Regularization or re-meshing introduces resolution dependent numerical diffusion, which has been shown to have a negligible effect on the overall accuracy of the simulations for large Reynolds number flows only when high resolution (40,000 particles for example) is used. It has been demonstrated that the re-meshed SPH is capable of DNS quality simulations. The method has been applied successfully to solve problems such as heat conducting flows [48], chemically reactive flows [49], and virtual surgery [50]. Yet, it is still an open problem how to design suitable regularization techniques in the presence of free surface, which is linked to the loss of adaptivity by reg- 
ularization. Another method of the second kind is the particle management technique [51], which partly modifies the particle distribution by merging particles too close to each other and inserting new particles into large holes and gaps. This requires on the one hand efficient detection procedures for nearby particles and large voids, on the other hand correct interpolation of the field data, when particles are merged and inserted. The particle management technique was implemented in LFPM.

Many previous works using SPH methods for simulations of incompressible flows adopted the approach of Monaghan [6], in which the real fluid is approximated by an artificial fluid which is weakly compressible. The artificial fluid has a specially designed equation of state with a speed of sound chosen to be still much larger than the speed of bulk flow and therefore has density fluctuations controlled to be small enough. Although simple for numerical implementation, weakly compressible SPH methods encounter problems such as sound wave reflection at boundaries, a stringent CFL time step constraint due to the high sound speed, and spurious pressure oscillations. To remedy these problems, strictly incompressible SPH (ISPH) methods have been developed [52-55], in which functions and their derivatives are still calculated by the SPH particle approximations. As mentioned before, the SPH particle approximations suffer the problem of inconsistency. If only a discrete velocitydivergence-free condition is enforced, larger density variation or particle clustering may occur and the density errors can accumulate during long time simulations. It was reported [56] that degradation and instability may happen in computations using ISPH for some simple viscous flows such as lid-driven cavity flow. Recently, Hu and Adams [57] proposed a fractional time-step SPH method to solve the difficulties concerning particle clustering and density error accumulation by enforcing both the zero-density-variation condition and the velocity-divergence-free condition at each full time step. A zero-density variation condition similar to that used in [57] was developed previously by Koshizuka et al. [58,59] for another incompressible meshless method, the socalled moving particle semi-implicit method. Ellero et al. [60] developed an alternative zero-density variation method by requiring that the volume of the fluid particles is constant and using Lagrangian multipliers to enforce the constrain. In LFPM [38,39], the Chorin's projection method [61] for solving the incompressible Navier-Stokers equation using grid-based methods is extended to the Lagrangian and meshless framework with the help of the weighted least squares method.

In this paper, a regularized Lagrangian finite point method for numerical simulation of incompressible viscous flows is proposed. A Lagrangian finite point scheme similar to that proposed by Tiwari and Kuhnert $[38,39]$ is applied to the projection method for the incompressible Navier-Stokes equations. The approximation of spatial derivatives is obtained by the weighted least squares method. Different from the work of Tiwari and Kuhnert $[38,39]$, the pressure 
Poisson equation with Neumann boundary condition is solved by a stabilized finite point method and the re-meshing technique is adopted to circumvent the problems associated with irregular particle distribution. Not like RSPH [48], the re-initialized particle distribution is not restricted to be uniform and the weighted least squares approximation is implemented to interpolate the properties of the old particles onto the new particle locations. The proposed regularized Lagrangian finite particle method (RLFPM) is tested against analytical and other numerical solutions for three benchmark problems demonstrating the capability of RLFPM as a meshless method for accurate and robust simulation of incompressible viscous flows.

The outline of the paper is as follows. In the next section, we introduce the partial differential equations (PDEs) governing the incompressible flow of a viscous fluid. In section 3, we describe the proposed methodology for solving the governing equations including the projection method, the weighted least squares method, the stabilized finite point method, and the re-meshing procedure. In section 4, numerical tests are presented. The paper ends up with concluding remarks in section 5 .

\section{Governing equations}

In the Lagrangian form, the governing equations for the motion of a viscous incompressible fluid are

$$
\begin{gathered}
\frac{\partial v_{\alpha}}{\partial x_{\alpha}}=0 \\
\rho \frac{D v_{\alpha}}{D t}=-\frac{\partial p}{\partial x_{\alpha}}+\mu \frac{\partial}{\partial x_{\beta}}\left(\frac{\partial v_{\alpha}}{\partial x_{\beta}}\right)+\rho g_{\alpha},
\end{gathered}
$$

where $\rho$ denotes the fluid density , $t$ the time, $v_{\alpha}$ the $\alpha$-th component of the fluid velocity, $x_{\alpha}$ the $\alpha$-th component of the position vector, $p$ is the isotropic pressure, $\mu$ the viscosity and $g_{\alpha}$ the component of a body force such as gravity. The Einstein summation convention is used in this paper (the summation is taken over repeated indices). In Eq. (2), $D / D t$ is the material (total) derivative defined in a fixed Eulerian frame by

$$
\frac{D}{D t}=\frac{\partial}{\partial t}+v_{\beta} \frac{\partial}{\partial x_{\beta}}
$$

\section{Regularized Lagrangian finite point method}

The flow domain is discretized into a finite number of "particles", who serve as interpolation points and move according to the velocity field. The governing 
equations (1) and (2) also known as Navier-Stokes equations are discretized in time using a time-integration scheme and on each particle using the point collocation approach with an interpolation process over neighboring particles. The relevant physical quantities on each particle are then obtained by solving the discrete governing equations. In the following, we describe each aspect of the proposed methodology in details.

\subsection{The projection method}

We consider the projection method [61] to solve the equations (1) and (2) in time. This is a semi-implicit method and is of first order accuracy in time. It consists of two fractional steps. At the first step the new position and the intermediate velocity for each particle are computed as follows:

$$
\begin{gathered}
x_{\alpha}^{n+1}=x_{\alpha}^{n}+\Delta t v_{\alpha}^{n} \\
v_{\alpha}^{*}=v_{\alpha}^{n}+\Delta t \frac{\mu}{\rho} \frac{\partial}{\partial x_{\beta}}\left(\frac{\partial v_{\alpha}^{n}}{\partial x_{\beta}}\right)+\Delta t g_{\alpha} .
\end{gathered}
$$

Eq. (5) is obtained by integrating the momentum equation forward in time without the pressure gradient term and using a first-order Euler scheme with an explicit treatment of the viscous term. Then, at the second step, $v_{\alpha}^{*}$ is corrected to give the new velocity $v_{\alpha}^{n+1}$ as

$$
v_{\alpha}^{n+1}=v_{\alpha}^{*}-\frac{\Delta t}{\rho} \frac{\partial p^{n+1}}{\partial x_{\alpha}},
$$

where the pressure gradient is added. Meanwhile, the new velocity must satisfy the incompressibility constraint

$$
\frac{\partial v_{\alpha}^{n+1}}{\partial x_{\alpha}}=0
$$

By taking the divergence of equation (6) and using the constraint equation

(7) for ensuring that $v_{\alpha}^{n+1}$ is a divergence free vector, we obtain the Poisson equation for the pressure

$$
\frac{\partial}{\partial x_{\alpha}}\left(\frac{\partial p^{n+1}}{\partial x_{\alpha}}\right)-\frac{\rho}{\Delta t} \frac{\partial v_{\alpha}^{*}}{\partial x_{\alpha}}=0
$$

Projecting the equation (6) on the outward unit normal vector $\boldsymbol{n}$ of the boundary $\Gamma$, we obtain the Neumann boundary condition for $p$, i.e,

$$
\left(\frac{\partial p}{\partial \boldsymbol{n}}\right)^{n+1}=-\frac{1}{\Delta t}\left[v_{\alpha}^{n+1} n_{\alpha}-v_{\alpha}^{*} n_{\alpha}\right]_{\Gamma} .
$$


Assuming $\boldsymbol{v} \cdot \boldsymbol{n}=0$ (non-penetration) on $\Gamma$, we get

$$
\left(\frac{\partial p}{\partial \boldsymbol{n}}\right)^{n+1}-\left[\frac{\mu}{\rho} \frac{\partial}{\partial x_{\beta}}\left(\frac{\partial v_{\alpha}^{n}}{\partial x_{\beta}}\right) n_{\alpha}+g_{\alpha} n_{\alpha}\right]_{\Gamma}=0
$$

on $\Gamma$.

In this Lagrangian projection method, the particle positions change only in the first step. The intermediate velocity, the pressure and the final divergence free velocity are all computed on the new particle positions. The spatial derivatives appearing in the above equations are approximated by the weighted least squares method. The Poisson pressure equation (8) with the Neumann boundary condition (10) is solved by a stabilized finite point method. The remeshing technique is adopted to avoid the problems associated with irregular particle distribution. As a whole, we name the proposed numerical method as the regularized Lagrangian finite point method (RLFPM).

\subsection{The weighted least squares method}

In general, we would like to approximate spatial derivatives of a function $f(\boldsymbol{x})$ in the computational domain $\Omega$. The problem is that we know only discrete function values exactly at the particles positions $\boldsymbol{x}_{i} \in \Omega, i=1, \ldots, N$, where $N$ is the total number of discretization points. To approximate a function derivative at a given point $\boldsymbol{x}$, only the discrete function values at the neighbor particles being in the support domain of $\boldsymbol{x}$ (a ball in 3D or a disk in 2D) should be taken into account. The weighted least squares (WLS) method can be applied for that purpose. The method does not require regular distribution of points. This is an advantage of this method. In the following, we explain the weighted least squares method in 2D (extension to 3D is straightforward).

Consider a Taylor's expansion of $f\left(\boldsymbol{x}_{i}\right)$ around $\boldsymbol{x}$

$f\left(\boldsymbol{x}_{i}\right)=f(\boldsymbol{x})+\sum_{\alpha=1}^{2} f_{\alpha}(\boldsymbol{x})\left(x_{i \alpha}-x_{\alpha}\right)+\frac{1}{2} \sum_{\alpha, \beta=1}^{2} f_{\alpha \beta}(\boldsymbol{x})\left(x_{i \alpha}-x_{\alpha}\right)\left(x_{i \beta}-x_{\beta}\right)+e_{i}$,

where $e_{i}$ is the truncation error in the Taylor's series expansion (here only to second-order, higher order expansions are, of course, possible), $f_{\alpha}$ is the derivative with respect to $x_{\alpha}$ (the $\alpha$-th component of the particle position vector $\boldsymbol{x}$ ) and $f_{\alpha \beta}$ the derivative with respect to $x_{\alpha}$ and $x_{\beta}$. The symbols $x_{i \alpha}$ and $x_{i \beta}$ denote the $\alpha$-th and $\beta$-th components of the particle position vector $\boldsymbol{x}_{i}$ respectively. From the given function values $f(\boldsymbol{x})$ and $f\left(\boldsymbol{x}_{i}\right)(i=1,2, \ldots, n)$, the unknowns $f_{\alpha}$ and $f_{\alpha \beta}$ for $\alpha, \beta=1,2$ (note that $f_{\alpha \beta}=f_{\beta \alpha}$ ) are computed by minimizing the error $e_{i}$ for $i=1,2, \ldots, n$. Here $n$ is the number of neighbor particles inside the support domain of $\boldsymbol{x}$. 
Using the Taylor's expansion (11) repeatedly for $i=1,2, \ldots, n$, the system of equations for the five unknowns can be written as

$$
e=M a-b
$$

with

$$
\begin{gathered}
\boldsymbol{e}=\left[e_{1}, e_{2}, \ldots, e_{n}\right]^{\mathrm{T}}, \\
\boldsymbol{a}=\left[f_{1}, f_{2}, f_{11}, f_{12}, f_{22}\right]^{\mathrm{T}}, \\
\boldsymbol{b}=\left[f\left(\boldsymbol{x}_{1}\right)-f(\boldsymbol{x}), f\left(\boldsymbol{x}_{2}\right)-f(\boldsymbol{x}), \ldots, f\left(\boldsymbol{x}_{n}\right)-f(\boldsymbol{x})\right]^{\mathrm{T}}, \\
M=\left[\boldsymbol{p}_{1}, \boldsymbol{p}_{2}, \ldots, \boldsymbol{p}_{n}\right]^{\mathrm{T}}
\end{gathered}
$$

where $\boldsymbol{a}$ is the vector containing the five unknowns and $M$ is a matrix in which the vector $\boldsymbol{p}_{i}$ is defined as

$$
\boldsymbol{p}_{i}=\left[x_{i 1}-x_{1}, x_{i 2}-x_{2}, \frac{\left(x_{i 1}-x_{1}\right)^{2}}{2},\left(x_{i 1}-x_{1}\right)\left(x_{i 2}-x_{2}\right), \frac{\left(x_{i 2}-x_{2}\right)^{2}}{2}\right]^{\mathrm{T}} .
$$

For $n>5$, this system is over-determined with respect to the five unknowns in $\boldsymbol{a}$. This problem can be simply overcome by determining the unknown vector $\boldsymbol{a}$ by minimizing the quadratic form

$$
J=\sum_{i=1}^{n} w_{i} e_{i}^{2}
$$

where $w_{i}=w\left(\boldsymbol{x}_{i}-\boldsymbol{x}\right)$ is the value of a weight function $w$ at point $\boldsymbol{x}_{i}$. Standard minimization of $J$ with respect to $\boldsymbol{a}$ gives

$$
\boldsymbol{a}=C^{-1} A \boldsymbol{b}
$$

where

$$
\begin{gathered}
C=\sum_{i=1}^{n} w_{i} \boldsymbol{p}_{i} \boldsymbol{p}_{i}^{\mathrm{T}}, \\
A=\left[w_{1} \boldsymbol{p}_{1}, w_{2} \boldsymbol{p}_{2}, \ldots, w_{n} \boldsymbol{p}_{n}\right] .
\end{gathered}
$$

The weight function is usually built in such a way that it takes a unit value in the vicinity of the point $\boldsymbol{x}$ where the function derivatives are to be computed and vanishes outside the support domain of $\boldsymbol{x}$. In this paper, we use a Gaussian weight function of the following form

$$
w(r, h)= \begin{cases}\exp \left(-\epsilon r^{2} / h^{2}\right), & \text { if } r \leq h \\ 0, & \text { else }\end{cases}
$$

where $r=\left\|\boldsymbol{x}_{i}-\boldsymbol{x}\right\|$ and $\epsilon$ is a positive constant chosen to be equal to 6.3 in our computations. The size of the searching radius $h$ determines $n$, the number 
of neighboring particles around $\boldsymbol{x}$ to be used for WLS approximation. For consistency reasons some obvious restrictions are required, for example, in 2D there should be at least 5 particles and they should not be on the same line or on a circle. The "linked-list" algorithm [62] for searching the neighboring particles is adopted in this paper.

\subsection{The stabilized finite point method for the Poisson equation}

We want to solve the pressure Poisson equation (8) with the Neumann boundary condition (10) in a meshless structure. We consider the stabilized finite point method proposed by Boroomand et al. [41]. A key component of the method is the stabilization of the Neumann boundary condition (see [41] for a detailed description). That is, instead of solving the original equations (8) and (10), the following equations must be solved for points inside or at the boundary of the domain:

$$
\begin{aligned}
{\left[\mathbb{A}\left(p^{n+1}\right)\right]_{i} } & =0 & & \text { for } i \in \text { inside points } \\
{\left[\mathbb{B}\left(p^{n+1}\right)\right]_{i}-\frac{1}{2} \gamma h_{n}\left[\mathbb{A}\left(p^{n+1}\right)\right]_{i} } & =0 & & \text { for } i \in \text { boundary points }
\end{aligned}
$$

where $\mathbb{A}$ and $\mathbb{B}$ represent the operators defined by the left hand sides of the equations (8) and (10) respectively, $\gamma$ is a suitable factor and $h_{n}$ a characteristic length. In our studies, $\gamma=0.5$ or 1 was found to give satisfactory results. The characteristic length $h_{n}$ is determined by the following expression

$$
h_{n}=2\left(A_{1}+A_{2}\right) /\left(L_{1}+L_{2}\right),
$$

where $A_{1}$ and $A_{2}$ are the areas associated to the boundary point $i$ and its nearest neighboring point inside the domain as shown in Figure 1, and $L_{1}$ and $L_{2}$ are their projections on the boundary.

Again, the spatial derivatives appearing in (19) are approximated by the weighted least squares method described in the previous subsection. The Dirichlet boundary condition is satisfied by simply prescribing the pressures on the corresponding boundary points to the fixed values. The final sparse linear algebraic equations for the unknown pressure values at the present time level $n+1$ are solved by an iterative scheme known as the preconditioned biconjugate gradient method [63]. At the beginning of the iteration, the initially guessed pressure values are taken as those from the previous time level $n$. If the pressures at the previous time step are close to those of the present time step, a few number of iterations is required to obtain a converged solution. It is also necessary to give the initial pressure value for each particle at time $t=0$. 


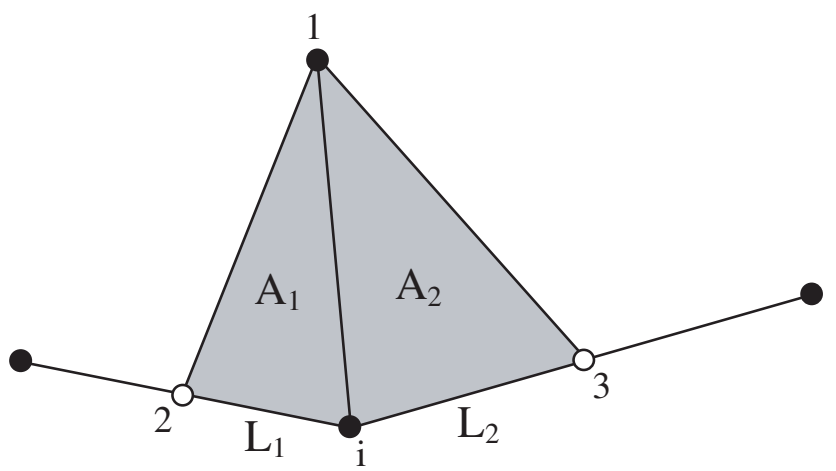

Fig. 1. Evaluation of the characteristic length $h_{n}$ for Neumann boundary points: point 1 is the closest inside point to master point $i, 2$ and 3 are the middle points between the master point and its two neighboring boundary points, $L_{1}$ and $L_{2}$ are the projections of constructed triangles $A_{1}$ and $A_{2}$ to the boundary of the domain.

\subsection{Regularization of particle distribution by re-meshing}

In the simulation procedure proposed in the subsection 3.1, particles move with the fluid velocity. Since the velocity field is the solution itself, one cannot predict in advance what the particle distribution will be. Hence, even if the particles are well distributed initially, they will in general cluster in some regions of the flow field and become insufficient in others. The former can in best case result in an unnecessarily high local resolution and thus computational effort, in the worst case yield numerical instabilities. The latter can spoil the accuracy and cause the problem of ill conditioned matrix in WLS. We use the re-meshing technique to circumvent these problems associated with irregular particle distribution created by the flow strain. In this technique, the position of the particles is periodically re-initialized to give a regular but not necessarily uniform distribution and the properties of the old particles are interpolated onto the new particle locations. This kind of technique has been implemented in a number of particle methods [64] including RSPH [48] but its application has not been reported before, to the best of our knowledge, in the context of FPM.

Various interpolation schemes may be applied for the re-meshing purpose. The simplest approach is to assign the arithmetic mean value of all field quantities at the closest neighboring old points to the new master point. This approach works well in 1d. In higher space dimensions, it is more stable to incorporate more than the closest neighbors into the interpolation. For particle methods such as vortex methods, interpolation formulas based on the integral representation of function are frequently used with a wide choice of interpolation or kernel functions [64]. They were also implemented in RSPH [48] due to their advantage of conserving the interpolated quantity. Since exact conservation is no more a concern in FPM, the choice made for this work is based on the 
second order WLS approximation, which has been found to yield satisfying results.

We start again from the Taylor's expansion (11) given in the subsection 3.2. The only difference from there is that $f(\boldsymbol{x})$ now represents the unknown function value at the new point $\boldsymbol{x}$, which has to be determined together with other unknowns $f_{\alpha}$ and $f_{\alpha \beta}$. We can write the system of equations in the same form as (12) but with

$$
\boldsymbol{a}=\left[f, f_{1}, f_{2}, f_{11}, f_{12}, f_{22}\right]^{\mathrm{T}}
$$

and

$$
\boldsymbol{b}=\left[f\left(\boldsymbol{x}_{1}\right), f\left(\boldsymbol{x}_{2}\right), \ldots, f\left(\boldsymbol{x}_{n}\right)\right]^{\mathrm{T}} .
$$

The vector $\boldsymbol{p}_{i}$ is now defined as

$$
\boldsymbol{p}_{i}=\left[1, x_{i 1}-x_{1}, x_{i 2}-x_{2}, \frac{\left(x_{i 1}-x_{1}\right)^{2}}{2},\left(x_{i 1}-x_{1}\right)\left(x_{i 2}-x_{2}\right), \frac{\left(x_{i 2}-x_{2}\right)^{2}}{2}\right]^{\mathrm{T}} \text {. }
$$

By using the same WLS approach as described in the subsection 3.2, we finally obtain the unknown vector $\boldsymbol{a}$ with the same expression as (15). The first component of $\boldsymbol{a}$ is the interpolated function value at the new point, which is looked for. The weight function $w$ and the number of neighboring old points $n$ play important roles in the interpolation. In our simulations, we found that the choice of $n=9$ and the same weight function (18) as used for approximating spatial derivatives gives the best results in terms of accuracy and stability.

Re-meshing can be performed with a frequency depending on the strain of the flow map. Since the projection method consists of two fractional steps, re-meshing can be implemented after either the first or the second fractional step. In this paper, re-meshing is performed after the first fractional step. In the case of regularization performed at every full time step, extra diffusion introduced by re-meshing can be quite large. In order to avoid unnecessary re-meshing when a very small time step is used or the strain of the flow is very low, we here introduce the maximum distance criterion for re-meshing, i.e., re-meshing is performed only when

$$
d^{\max } \geq \xi \Delta d
$$

where $d^{\max }$ is the maximum of distances between new particle positions and previously re-initialized particle positions, $\Delta d$ the nearest neighbor distance in the previously re-initialized particle configuration, and $\xi$ a parameter typically set to 0.25 . We will show later in the subsection 4.1 that the maximum distance criterion helps to reduce the extra diffusion error significantly. The re-initialized particle distribution can vary at different time levels, which is essential for the simulation of flow problems with time-varying boundaries such as free surface flows. An example will be given in the subsection 4.2. 


\subsection{Time step selection}

Since the numerical scheme is essentially explicit, several time step constraints must be satisfied for numerical stability, including a Courant-Friedrichs-Lewy (CFL) condition,

$$
\Delta t \leq 0.15 \frac{h}{U_{\max }}
$$

and a constraint due to viscous diffusion,

$$
\Delta t \leq 0.125 \frac{h^{2}}{\nu}
$$

where $U_{\max }$ is the maximum value of velocity for a given problem and $\nu=\mu / \rho$ is the kinematic viscosity. If a body force is to be considered, we then have the additional constraint

$$
\Delta t \leq 0.25 \min _{\forall a} \sqrt{\frac{h}{g_{\alpha}}}
$$

\section{Numerical examples}

In this section, we examine the validity of RLFPM proposed in this paper for the numerical simulation of incompressible viscous flows. Three benchmark two-dimensional problems are considered. They are Taylor-Green flow, lid-driven flow in a cavity and flow through a periodic lattice of cylinders. For the first problem, we have compared the numerical solutions obtained by RLFPM with the exact ones and analyzed spatial and temporal convergence of RLFPM. The problem of extra diffusion due to remeshing is discovered for large Reynolds numbers. For the second problem, our numerical solutions have been compared with solutions obtained by a finite difference method and a very good agreement of both solutions has been found. In the third problem, we find that RLFPM results are in close agreement with results from a finite element method and better than those from a weakly compressible SPH method.

\subsection{Taylor-Green flow}

As a first test of RLFPM, we perform a simulation of two-dimensional TaylorGreen flow. The Taylor-Green flow is a periodic array of decaying vortices in the $(x, y)$ plane with velocity components given by

$$
v_{x}(x, y, t)=-U e^{b t} \cos (2 \pi x) \sin (2 \pi y),
$$




$$
v_{y}(x, y, t)=U e^{b t} \sin (2 \pi x) \cos (2 \pi y)
$$

where $b=-8 \pi^{2} /$ Re. The Reynolds number here is defined as

$$
\operatorname{Re}=\frac{U L}{\nu},
$$

where $L$ is the characteristic length. The computation is performed in a periodic domain $[0, L] \times[0, L]$ with $L=1 \mathrm{~m}$. The initial particle velocity is assigned according to Eqs. (25) and (26) with $t=0$ and $U=1 \mathrm{~ms}^{-1}$. The initial pressure is given as $p(x, y, 0)=0$. The computational domain was modeled with $N \times N$ real particles which were placed with a uniform spacing of $\Delta x=\Delta y$. During the simulation, the re-initialized particle distribution was kept the same as the original one. The searching radius $h$ was set to $3 \Delta x$ and the stabilization factor $\gamma$ was assigned to 1 . The periodic boundary conditions in all directions are enforced by placing imaginary particles outside the calculation region. For example, let $d$ be the horizontal distance of a real particle to the left boundary at $x=0$, if $d \leq h$, then a imaginary particle is placed at $x=L+d$ with other properties including the $y$ coordinate copied from the corresponding real particle.

For the error analysis of the RLFPM simulations, we used the relative error

$$
L_{\infty}=\max _{t=0}^{T}\left(\left|\frac{u_{e x}^{t}-u^{t}}{u_{e x}^{t}}\right|\right),
$$

where $u_{e x}^{t}$ denotes the maximum velocity of the exact solution at time $t$ and $u^{t}$ the maximum velocity of the numerical simulation at time $t$. For the analysis of spatial convergence, $T$ is the time where $u_{e x}^{T}=U / 50$, which is the same as used for RSPH [48]. For the analysis of temporal convergence, $T$ is the time where $u_{e x}^{T}=U / 5$. Figure 2 shows the relative error $\left(L_{\infty}\right)$ as a function of the number of particles for the RLFPM and RSPH calculations at $\mathrm{Re}=1$. The time step is fixed to $5 \times 10^{-5} \mathrm{~s}$ for the RLFPM calculations. For both RLFPM and RSPH, the relative error decreases as the number of particles is increased. The convergence rate of RLFPM is faster than that of RSPH. When the number of particles is larger than 3600, RLFPM $\left(L_{\infty}<1 \%\right)$ is more accurate than RSPH $\left(L_{\infty}<3 \%\right)$. Figure 3 gives the temporal convergence plot of RLFPM for $R e=1$ with the number of particles fixed to 3600 . An intriguing finding is that the error of RLFPM decreases as the time step increases in a certain range. Nevertheless, the same feature has been already discovered in a similar mesh-based method, semi-Lagrangian method [65]. Falcone and Ferretti [66] showed that the overall error of semi-Lagrangian method is indeed not monotonic with respect to time step $\Delta t$ and it has the particular form:

$$
\mathcal{O}\left(\Delta t^{k}+\frac{\Delta x^{p+1}}{\Delta t}\right)
$$

where $k$ refers to the order of time integration and $p$ to the interpolation order. 


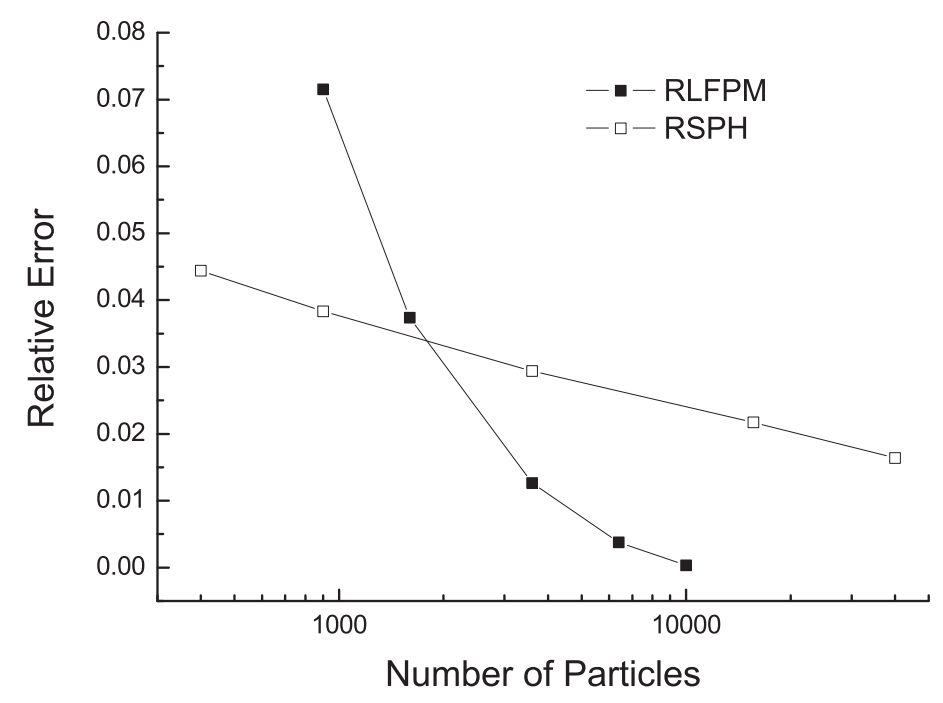

Fig. 2. Relative error of RLFPM and RSPH simulations of the Taylor-Green flow at $\mathrm{Re}=1$ with different spatial resolutions.

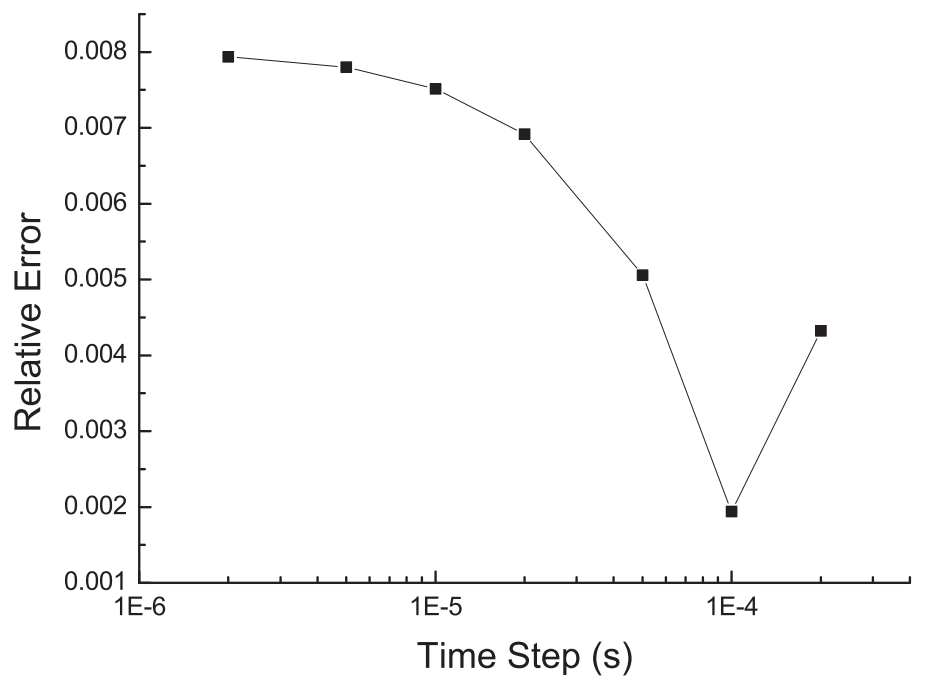

Fig. 3. Relative error of RLFPM simulations of the Taylor-Green flow at Re $=1$ with different temporal resolutions.

It can be expected that the overall error form of RLFPM with respect to time step possesses a similar structure as above. The time-depended behavior of the maximum velocity of the flow for $\mathrm{Re}=1000$ calculated from RLFPM with different options is presented in Fig. 4 and is compared to the analytical solution. With a medium resolution (3600 particles), the RLFPM solution with remeshing performed at every time step $\left(L_{\infty}<24 \%\right)$ decays much faster than the exact solution does. This numerical hyperviscous effect is due to extra diffusion introduced by remeshing, which can be quite large in the case of regularization performed at every time step. It can be dominant in the simulations of large Reynolds number flows as shown in this example. By performing remeshing according to the maximum distance criterion, the effect of extra diffusion is reduced and the overall accuracy is improved to $L_{\infty}<10 \%$ 


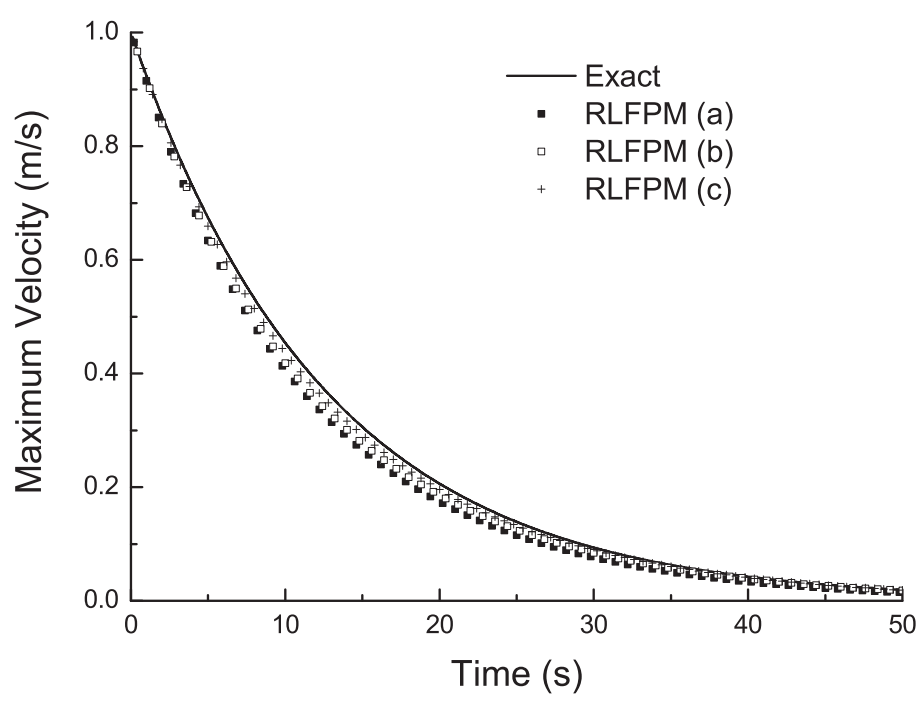

Fig. 4. Decay of the maximum velocity for $R e=1000$. Comparison of the exact solution with (a) the RLFPM solution with 3600 particles and remeshing performed at every time step, (b) the RLFPM solution with 3600 particles and remeshing performed according to the maximum distance criterion, and (c) the RLFPM solution with 10000 particles and remeshing performed according to the maximum distance criterion.

with the same resolution (3600 particles). To remain the simulations accurate enough for large Reynolds numbers $(\mathrm{Re}=1000)$, it is necessary to increase the particle resolution, e.g., the RLFPM solution using 10000 particles yields $L_{\infty}<5 \%$ (Fig. 4), which is the level of accuracy achieved by the RSPH simulation using 40000 particles. An antidiffusive or high-order interpolation scheme may be implemented into the remeshing process to minimize the error and further improve the overall accuracy.

The CPU cost per full time step in a Pentium $4 \mathrm{PC}$ is $1.8 \mathrm{~s}$ for 3600 particles and $6.4 \mathrm{~s}$ for 10000 particles. The CPU cost per particle in the latter case is higher than that in the former case because computational cost for solving the pressure Poisson equation does not scale linearly with the number of particles.

\subsection{Lid-driven flow in a cavity}

The second test case is the flow in a closed square due to a horizontal moving of the top side of the square while the other three sides remain stationary. The flow will form a recirculation pattern after reaching steady state. This is a classic benchmark simulation and steady flow in a cavity has become a popular example for testing and comparing numerical methods for many years. The dimension of the side of the square domain is considered to be $L=1 \mathrm{~m}$. Most of the simulations in literature are performed with constant horizontal velocity over the top side. Because of the velocity discontinuity at 


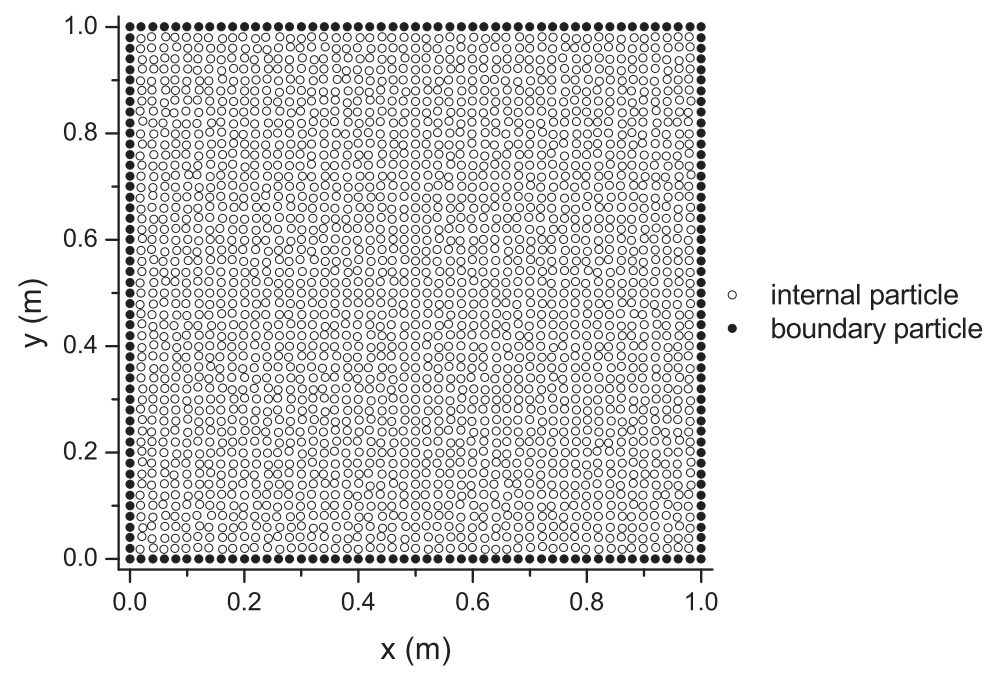

Fig. 5. Initial and re-initialized particle distribution used in the lid-driven cavity flow simulation.

the top two corners, the solution of the Navier-Stokes equations is in some way "singular" at these points (the vorticity becomes infinite). As a consequence, it is difficult to compare solutions since the approximation of the flow close to the singularity affects the accuracy of the solution. To avoid such a singularity, we consider a regular solution of the problem suggested by Tiwari and Kuhnert [67], who proposed to prescribe the velocity on the top side of the cavity as

$$
v_{x}(x, 1)=16 x^{2}(1-x)^{2}, v_{y}(x, 1)=0 .
$$

Note that the horizontal velocity is not longer constant. Homogeneous Dirichlet boundary conditions $v_{x}=v_{y}=0$ are applied over the rest of the boundaries. The Neumann boundary condition (10) is applied on all the boundaries for solving the pressure Poisson equation.

A total of $2601(51 \times 51)$ particles were first evenly placed in equal distance $(\Delta x=\Delta y=0.02 \mathrm{~m}$ ) over the problem domain with 200 of them located right on the four sides of the square (51 on each side), which are so called boundary particles. Then, the positions of boundary particles were fixed, while the positions of internal particles were shifted with slight random displacements to give an arbitrary non-uniform particle distribution as shown in Fig. 5. The reason for doing this is to show that RLFPM is more flexible than FDM and RSPH. Again the searching radius $h$ was set to $3 \Delta x$ and the stabilization factor $\gamma$ was assigned to 1 . The density is set to $\rho=1 \mathrm{kgm}^{-3}$ and the characteristic velocity is chosen to be $U=1 \mathrm{~ms}^{-1}$. By changing the viscosity, simulations corresponding to four different Reynolds numbers were performed. Figure 6 and Figure 7 show the steady vertical velocity profile along the horizontal centerline of the square and the steady horizontal velocity profile along the vertical centerline of the square respectively for Reynolds numbers equal to 10, 100, 400 and 1000. The FDM results presented for comparison were ob- 


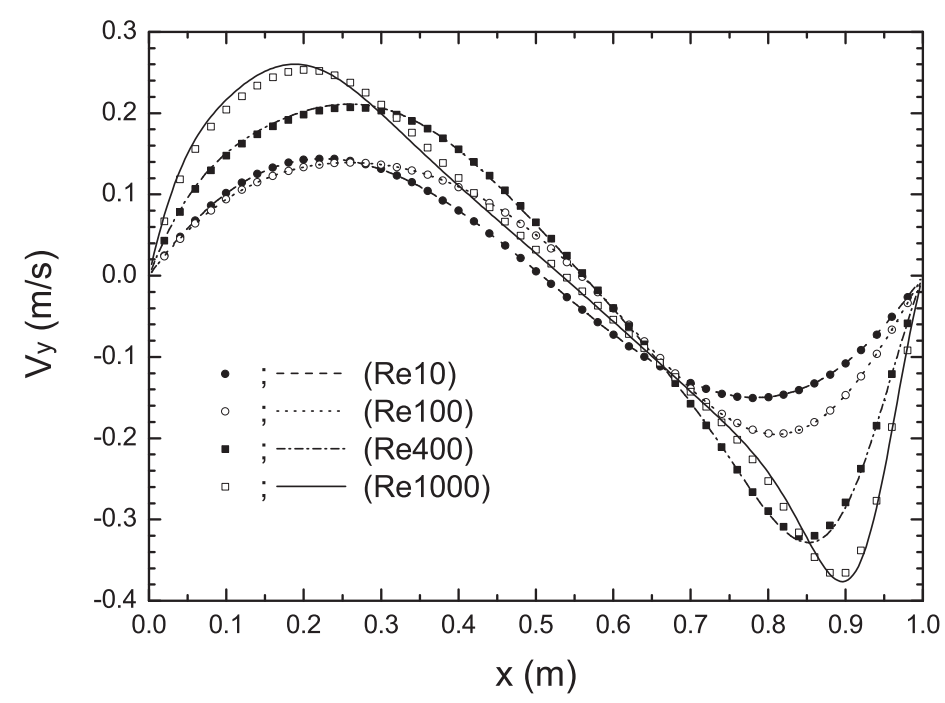

Fig. 6. Comparisons of the results obtained using RLFPM (symbols) and FDM (lines) for the lid-driven flow in a cavity: the steady vertical velocity profiles along the horizontal centerline of the square for Reynolds numbers 10, 100, 400 and 1000.

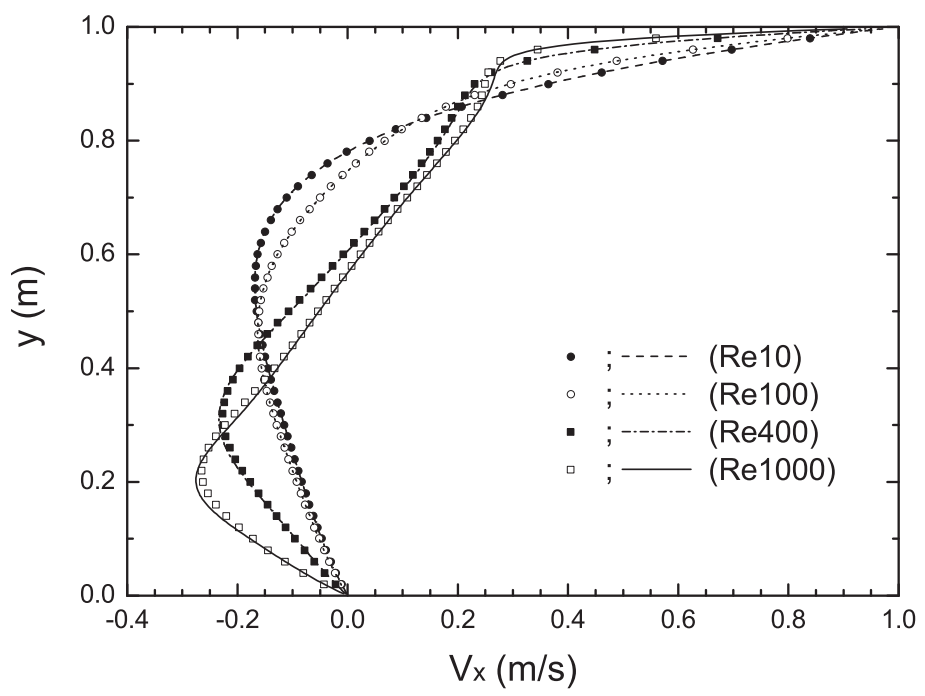

Fig. 7. Comparisons of the results obtained using RLFPM (symbols) and FDM (lines) for the lid-driven flow in a cavity: the steady horizontal velocity profile along the vertical centerline of the square for Reynolds numbers 10, 100, 400 and 1000.

tained by using a finite difference method to solve the Navier-Stokes equations in streamfunction and vorticity formulation with a fine grid of $301 \times 301$ (see [68] for details). It can be observed that the results obtained using RLFPM and FDM are almost identical in the lower Reynolds number cases and they are in good agreement in the higher Reynolds number cases. For all these simulations, in the re-meshing procedure, the re-initialized particle distribution was kept the same as the initial one. For the Reynolds number 400, we also performed the simulation by varying the re-initialized particle distribution at each re-meshing step and in a random way as described before. The simulation went well and no significant differences were observed between the results with 


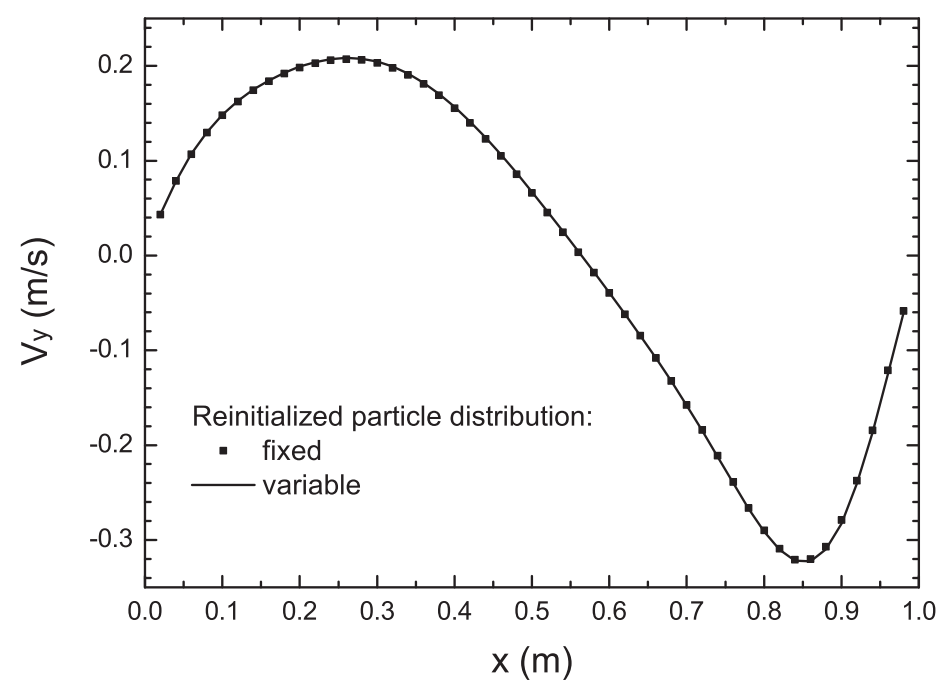

Fig. 8. Comparisons of the results obtained using RLFPM with fixed and variable re-initialized particle distribution for the lid-driven flow in a cavity: the steady vertical velocity profiles along the horizontal centerline of the square for Reynolds number 400 .

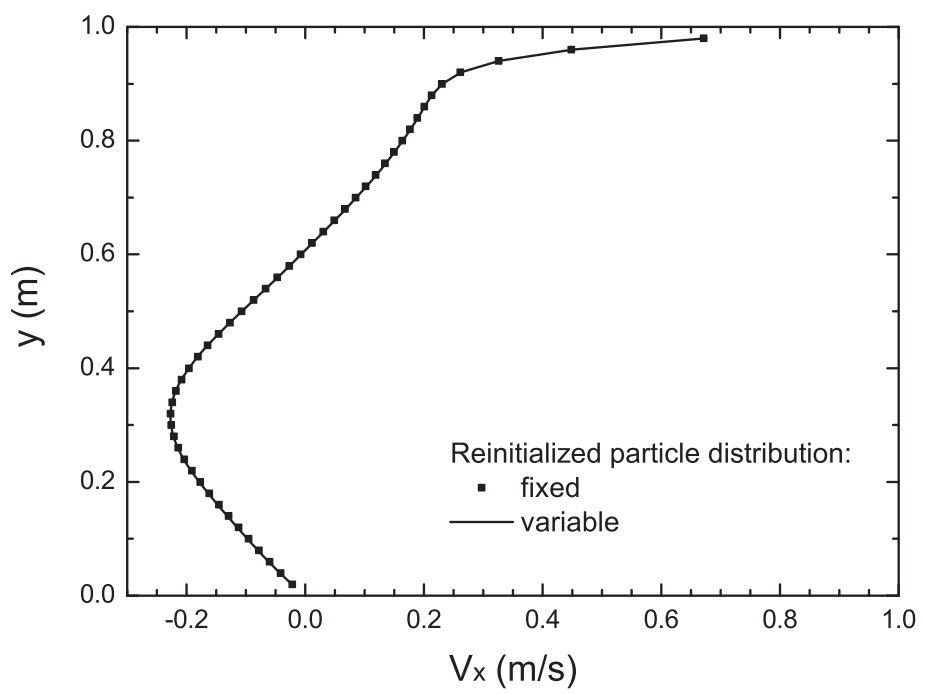

Fig. 9. Comparisons of the results obtained using RLFPM with fixed and variable re-initialized particle distribution for the lid-driven flow in a cavity: the steady horizontal velocity profile along the vertical centerline of the square for Reynolds number 400 .

variable re-initialization and those with fixed re-initialization (Fig. 8 and Fig. $9)$.

Finally, to check the convergence of RLFPM, we performed the simulation with a refined discretization using $10201(101 \times 101)$ particles for the case of $\operatorname{Re}=1000$. The results are shown in Figure 10 and Figure 11 for the steady vertical velocity profile along the horizontal centerline of the square and the steady horizontal velocity profile along the vertical centerline of the 


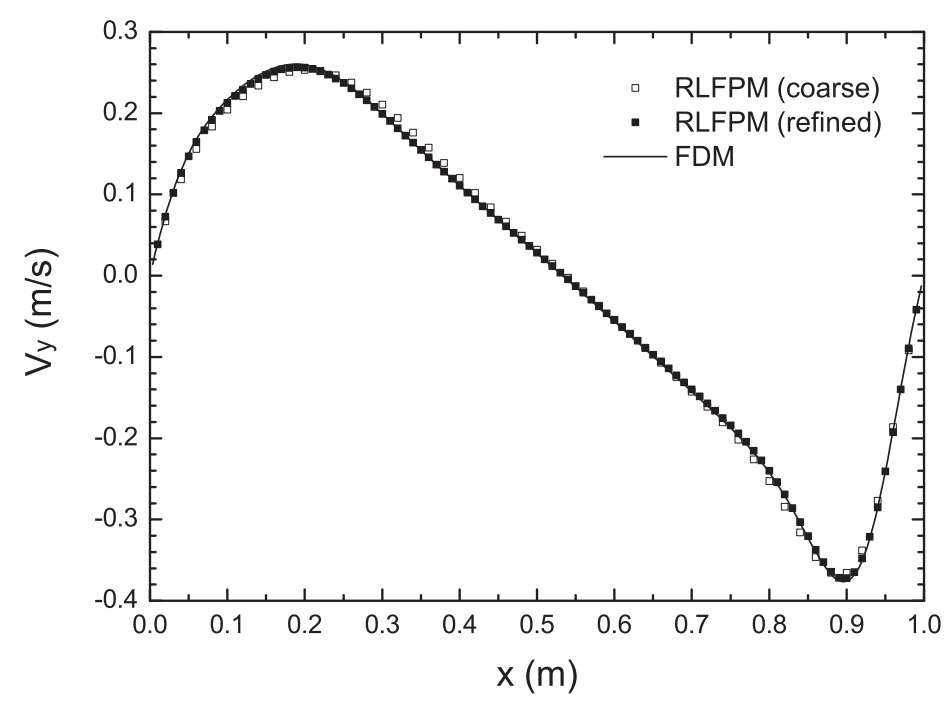

Fig. 10. Comparisons of the results obtained using RLFPM with coarse and refined discretization for the lid-driven flow in a cavity: the steady vertical velocity profiles along the horizontal centerline of the square for Reynolds number 1000.

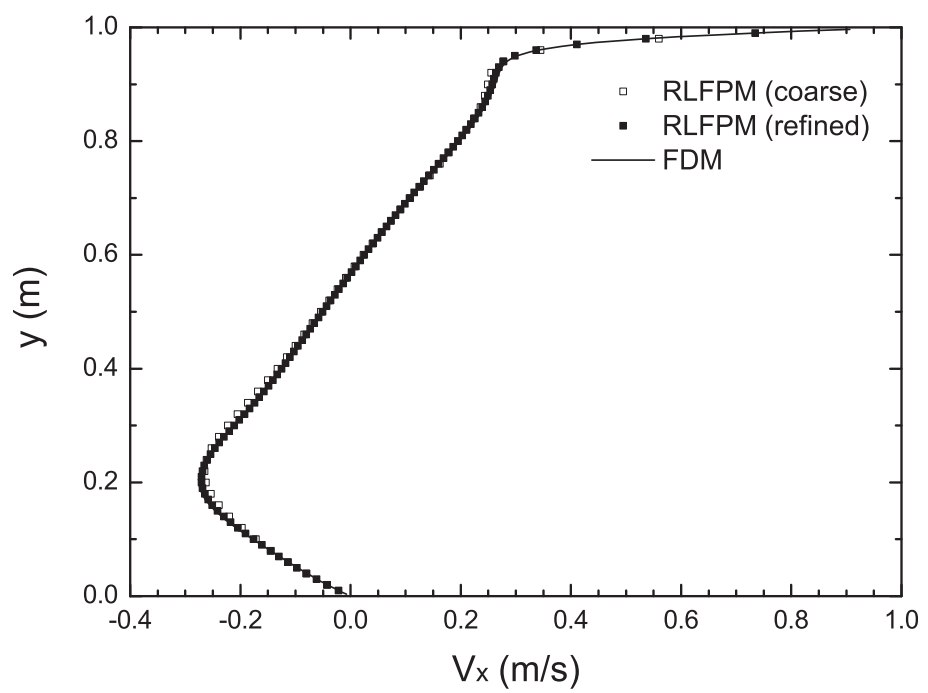

Fig. 11. Comparisons of the results obtained using RLFPM with coarse and refined discretization for the lid-driven flow in a cavity: the steady horizontal velocity profile along the vertical centerline of the square for Reynolds number 1000.

square respectively. It can be seen that the differences between the RLFPM results with coarse mesh and those with refined mesh are small, and the results obtained by RLFPM using the refined mesh $(101 \times 101)$ is almost overlapped with the results obtained by FDM using a very fine grid $(301 \times 301)$. 


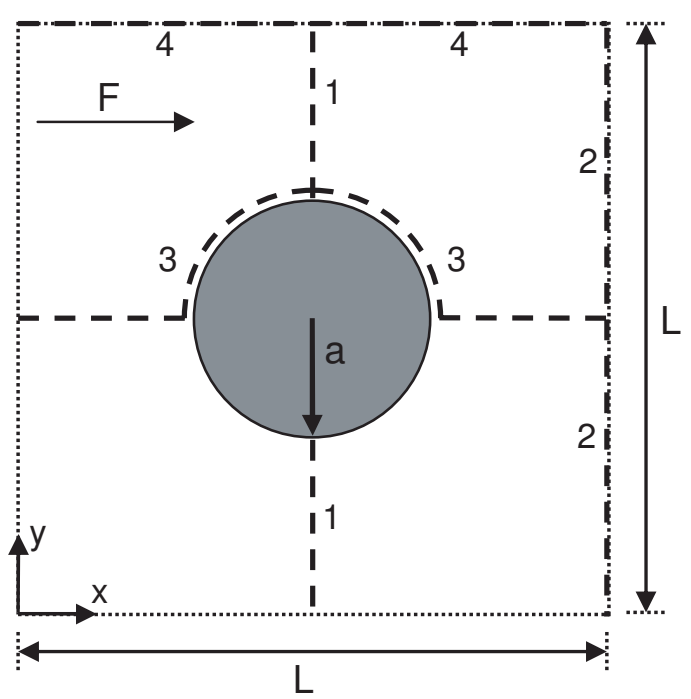

Fig. 12. Single cylinder within a periodic lattice and paths for comparison of numerical solutions.

\subsection{Flow through a periodic lattice of cylinders}

The third test of the proposed method involves flow through a square lattice of cylinders, which has been studied extensively as a simple model of flow through fibrous porous media. The computational domain of the problem consists of a single cylinder of radius $a$ and its associated volume within the lattice (see Fig. 12). Flow is driven by a body force along the $x$-direction $\left(g_{x}=F\right)$. On the four sides of the square, periodic boundary conditions are applied to model an infinite periodic arrangement of cylinders. On the boundary of the single cylinder, no-slip boundary condition is applied.

Numerical simulation of flow through a periodic lattice of cylinders was performed using RLFPM for $L=0.1 \mathrm{~m}, a=0.02 \mathrm{~m}, \nu=10^{-6} \mathrm{~m}^{2} \mathrm{~s}^{-1}$, and $F=1.5 \times 10^{-7} \mathrm{~ms}^{-2}$. Replacing $L$ with $a$ in Eq. (27) and choosing the characteristic velocity to be $U=5 \times 10^{-5} \mathrm{~ms}^{-1}$ gives $\mathrm{Re}=1$. The same problem has been also modelled using a weakly compressible SPH method (WSPH) and a finite element method (FEM) in [8]. The WSPH simulation used approximately 3000 particles. To be comparable with it, our simulation was run using 2264 particles with 50 of them being located on the perimeter of the cylinder. The nearest neighbor distance $\Delta x$ was about $0.002 \mathrm{~m}$ and the searching radius $h$ was set to $3 \Delta x$. The periodic boundary conditions on the four sides of the square are implemented by generating imaginary particles in the same way as in the first test case.

Steady velocity and pressure distributions for $\gamma=0.5$ and $\gamma=1$ are compared by plotting values along the four paths defined in Fig. 12 . The arc of path 3 was taken $0.002 \mathrm{~m}$ beyond the cylinder surface. Fig. 13 shows velocity profiles 


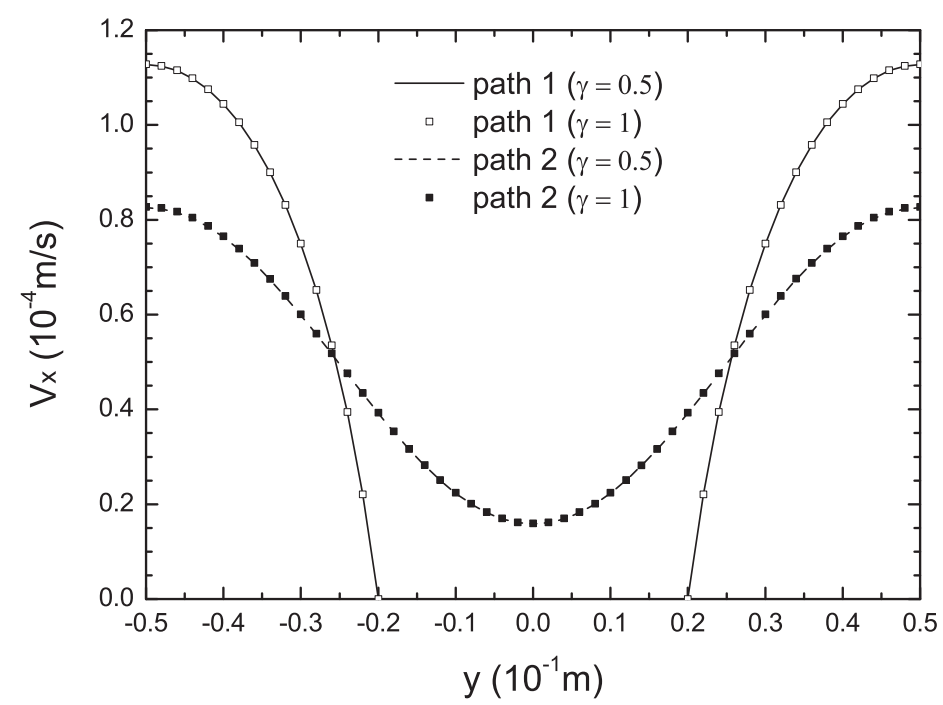

Fig. 13. Comparisons of velocity profiles along paths 1 and 2 obtained using RLFPM with $\gamma=0.5$ and $\gamma=1$.

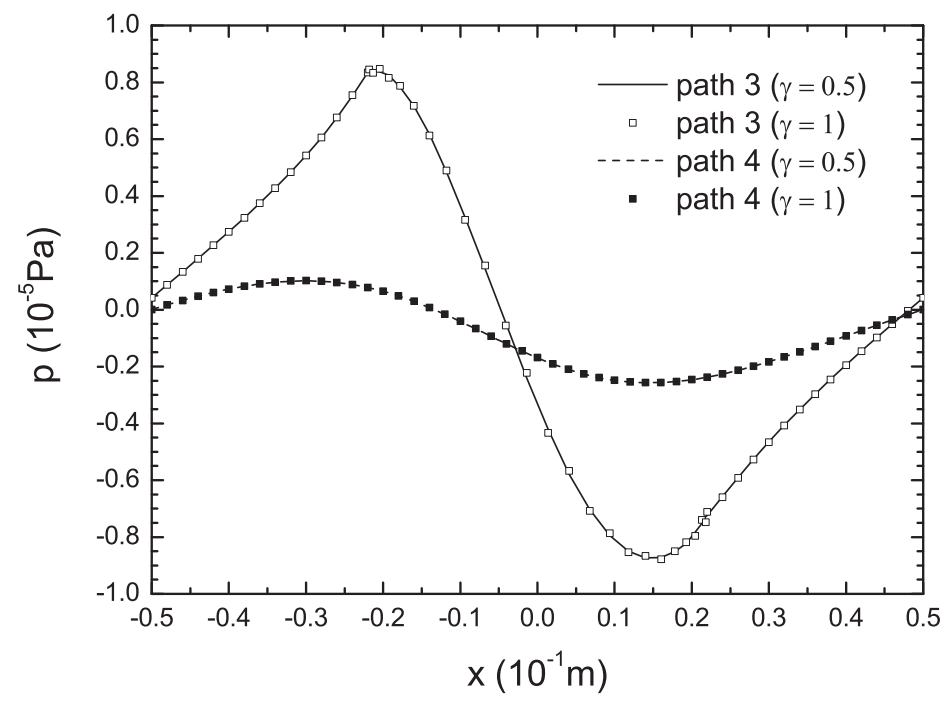

Fig. 14. Comparisons of pressure profiles along paths 3 and 4 obtained using RLFPM with $\gamma=0.5$ and $\gamma=1$.

obtained using RLFPM for path 1 and path 2. The differences between the results with $\gamma=0.5$ and the results with $\gamma=1$ are negligible. A direct visual comparison with the velocity profiles in Fig. 6 of [8] shows that the results obtained using RLFPM are in close agreement with those from the WSPH and the FEM throughout the flow domain. In Fig. 14, we plot the dynamic pressure profiles along paths 3 and 4 . There are only small differences between the predicted pressures with $\gamma=0.5$ and those with $\gamma=1$. The predicted pressure profile along path 3 with $\gamma=1$ shows small local fluctuations in the areas just before and after the cylinder, while that with $\gamma=0.5$ is fluctuation free. Again, through a direct visual comparison with the pressure profiles in Fig. 8 of [8], we find that the simulated pressure profiles using RLFPM are 
in good agreement with those simulated by the FEM and superior to those predicted by the WSPH which exhibit unphysical fluctuations.

\section{Conclusions}

A regularized Lagrangian finite point method (RLFPM) is proposed in this paper for the numerical simulation of incompressible viscous flows. Due to the adoption of the truly incompressible approach (the projection method), the proposed method is not subject to a severe restriction on time step and does not produce unphysical pressure oscillations. Therefore, it could be more efficient than other Lagrangian particle methods who use the weakly compressible approach, particularly as the Reynolds number is increased. Two key components of RLFPM are the application of the stabilized finite point method for solving the pressure Poisson equation with Neumann boundary condition and the implementation of the re-meshing technique for overcoming the problems associated with irregular particle distribution, which is encountered typically in Lagrangian particle methods. Numerical tests including Taylor-Green flow, lid-driven flow in a cavity and flow through a periodic lattice of cylinders are presented to validate the proposed methodology. The results indicate that RLFPM is an accurate and robust numerical method for the meshless solution of incompressible viscous flows. The accuracy and stability of RLFPM comes with a minimal additional computational cost due to re-meshing. However, with medium spatial resolutions and for large Reynolds number flows, extra diffusion caused by re-meshing has been shown to have a non-negligible numerical hyperviscous effect on the overall accuracy of the simulations, especially when re-meshing is performed at every time step. The maximum distance criterion for re-meshing can help to alleviate the effect of extra diffusion. To further reduce the extra diffusion error, it is necessary to increase the particle resolution or consider the implementation of an antidiffusive process.

\section{References}

[1] R. A. Gingold, J. J. Monaghan, Smoothed particle hydrodynamics: Theory and application to non-spherical stars, Mon. Not. Roy. Astron. Soc. 181 (1977) 375389.

[2] L. B. Lucy, A numerical approach to the testing of the fission hypothesis, Astron. J. 82 (12) (1977) 1013-1024.

[3] W. Benz, E. Asphaug, Simulations of brittle solids using smooth particle hydrodynamics, Comput. Phys. Commun. 87 (1-2) (1995) 253-265. 
[4] L. D. Libersky, A. G. Petschek, Smooth particle hydrodynamics with strength of materials, in: H. E. Trease, M. J. Fritts, W. P. Crowley (Eds.), Advances in the Free-Lagrange Method, Vol. 395 of Lect. Notes in Phys., Springer-Verlag, New York/Berlin, 1991, pp. 248-257.

[5] L. D. Libersky, A. G. Petschek, T. C. Carney, J. R. Hipp, F. A. Allahdadi, High strain lagrangian hydrodynamics a three-dimensional SPH code for dynamic material response, J. Comput. Phys. 109 (1) (1993) 67-75.

[6] J. J. Monaghan, Simulating free surface flows with SPH, J. Comput. Phys. 110 (2) (1994) 399-406.

[7] O. Flebbe, S. Münzel, H. Herold, H. Riffert, H. Ruder, Smoothed particle hydrodynamics: Physical viscosity and the simulation of accretion disks, Astrophys. J. 431 (2) (1994) 754-760.

[8] J. P. Morris, P. J. Fox, Y. Zhu, Modeling low reynolds number incompressible flows using SPH, J. Comput. Phys. 136 (1997) 214-226.

[9] H. Takeda, S. M. Miyama, M. Sekiya, Numerical simulation of viscous flow by smoothed particle hydrodynamics, Prog. Theor. Phys. 92 (5) (1994) 939-960.

[10] S. J. Watkins, A. S. Bhattal, N. Francis, J. A. Turner, A. P. Whitworth, A new prescription for viscosity in smoothed particle hydro dynamics, Astron. Astrophys. Suppl. Ser. 119 (1) (1996) 177-187.

[11] C. Maveyraud, W. Benz, A. Sornette, D. Sornette, Solid friction at high sliding velocities: An explicit three-dimensional dynamical smoothed particle hydrodynamics approach, J. Geophys. Res. B: Solid Earth 104 (B12) (1999) 28769-28788.

[12] P. W. Cleary, J. J. Monaghan, Conduction modelling using smoothed particle hydrodynamics, J. Comput. Phys. 148 (1) (1999) 227-264.

[13] J. J. Monaghan, A. Kocharyan, SPH simulation of multi-phase flow, Comput. Phys. Commun. 87 (1-2) (1995) 225-235.

[14] J. P. Morris, Simulating surface tension with smoothed particle hydrodynamics, Int. J. Numer. Methods Fluids 33 (2000) 333-353.

[15] R. Ata, A. Soulaïmani, A stabilized SPH method for inviscid shallow water flows, Int. J. Numer. Methods Fluids 47 (2) (2005) 139-159.

[16] P. W. Cleary, M. Prakash, Discrete-element modelling and smoothed particle hydrodynamics: Potential in the environmental sciences, Philos. Trans. R. Soc. A-Math. Phys. Eng. Sci. 362 (1822) (2004) 2003-2030.

[17] L. Oger, S. B. Savage, Smoothed particle hydrodynamics for cohesive grains, Comput. Meth. Appl. Mech. Eng. 180 (1-2) (1999) 169-183.

[18] J. J. Monaghan, SPH compressible turbulence, Mon. Not. Roy. Astron. Soc. 335 (3) (2002) 843-852. 
[19] M. Ellero, M. Kröger, S. Hess, Viscoelastic flows studied by smoothed particle dynamics, J. Non-Newton. Fluid Mech. 105 (1) (2002) 35-51.

[20] J. Fang, R. G. Owens, L. Tacher, A. Parriaux, A numerical study of the SPH method for simulating transient viscoelastic free surface flows, J. Non-Newton. Fluid Mech. 139 (1-2) (2006) 68-84.

[21] J. J. Monaghan, Smoothed particle hydrodynamics, Annu. Rev. Astron. Astrophy. 30 (1) (1992) 543-574.

[22] W. K. Liu, S. Jun, Y. F. Zhang, Reproducing kernel particle methods, Int. J. Numer. Methods Fluids 20 (8-9) (1995) 1081-1106.

[23] G. R. Johnson, S. R. Beissel, Normalized smoothing functions for SPH impact computations, Int. J. Numer. Methods Eng. 39 (16) (1996) 2725-2741.

[24] G. A. Dilts, Moving-least-squares-particle hydrodynamics - i. consistency and stability, Int. J. Numer. Methods Eng. 44 (8) (1999) 1115-1155.

[25] G. A. Dilts, Moving least-squares particle hydrodynamics ii: Conservation and boundaries, Int. J. Numer. Methods Eng. 48 (10) (2000) 1503-1524.

[26] J. K. Chen, J. E. Beraun, A generalized smoothed particle hydrodynamics method for nonlinear dynamic problems, Comput. Meth. Appl. Mech. Eng. 190 (1-2) (2000) 225-239.

[27] M. B. Liu, W. P. Xie, G. R. Liu, Modeling incompressible flows using a finite particle method, Appl. Math. Model. 29 (12) (2005) 1252-1270.

[28] G. M. Zhang, R. C. Batra, Modified smoothed particle hydrodynamics method and its application to transient problems, Comput. Mech. 34 (2) (2004) 137-146.

[29] J. Bonet, T. S. L. Lok, Variational and momentum preservation aspects of smooth particle hydrodynamic formulations, Comput. Meth. Appl. Mech. Eng. 180 (1-2) (1999) 97-115.

[30] J. Fang, A. Parriaux, M. Rentschler, C. Ancey, Improved SPH methods for simulating free surface flows of viscous fluids, Appl. Numer. Math. (2008), doi:10.1016/j.apnum.2008.02.003

[31] G. Oger, M. Doring, B. Alessandrini, P. Ferrant, An improved SPH method: Towards higher order convergence, J. Comput. Phys. 225 (2) (2007) 1472-1492.

[32] E. Oñate, S. Idelsohn, O. Zienkiewicz, R. Taylor, A finite point method in computational mechanics: Applications to convective transport and fluid flow, Int. J. Numer. Methods Eng. 39 (1996) 3839-3866.

[33] E. Oñate, S. Idelsohn, O. Zienkiewicz, R. Taylor, A stabilized finite point method for analysis of fluid mechanics problems, Comput. Meth. Appl. Mech. Eng. 139 (1996) 315-346.

[34] E. Oñate, S. Idelsohn, A mesh-free finite point method for advective-diffusive transport and fluid flow problems, Comput. Mech. 21 (1998) 283-292. 
[35] E. Oñate, S. Sacco, C. Idelsohn, A finite point method for incompressible flow problems, Comput. Visual. Sci. 3 (2000) 67-75.

[36] E. Oñate, F. Perazzo, J. Miquel, A finite point method for elasticity problems, Comput. Struct. 79 (2001) 2151-2163.

[37] R. Löhner, C. Sacco, E. Oñate, S. Idelsohn, A finite point method for compressible flow, Int. J. Numer. Methods Eng. 53 (8) (2002) 1765-1779.

[38] S. Tiwari, J. Kuhnert, Modeling of two-phase flows with surface tension by finite pointset method (FPM), J. Comput. Appl. Math. 203 (2007) 376-386.

[39] S. Tiwari, S. Antonov, D. Hietel, J. Kuhnert, F. Olawsky, R. Wegener, A meshfree method for simulations of interactions between fluids and flexible structures, in: M. Griebel, M. Schweitzer (Eds.), Meshfree Methods for Partial Differential Equations III, Vol. 57 of Lecture Notes in Computational Science and Engineering, Springer, Berlin, 2007, pp. 249-264.

[40] E. Oñate, Derivation of stabilized equations for numerical solution of advectivediffusive transport and fluid flow problems, Comput. Meth. Appl. Mech. Eng. 151 (1-2) (1998) 233-265.

[41] B. Boroomand, A. A. Tabatabaei, E. Oñate, Simple modifications for stabilization of the finite point method, Int. J. Numer. Methods Eng. 63 (3) (2005) 351-379.

[42] J. M. Owen, J. V. Villumsen, P. R. Shapiro, H. Martel, Adaptive smoothed particle hydrodynamics: Methodology. II, Astrophys. J. Suppl. Ser. 116 (2) (1998) 155-209.

[43] P. R. Shapiro, H. Martel, J. V. Villumsen, J. M. Owen, Adaptive smoothed particle hydrodynamics, with application to cosmology: Methodology, Astrophys. J. Suppl. Ser. 103 (2) (1996) 269-330.

[44] R. Vignjevic, J. R. Reveles, J. Campbell, SPH in a total Lagrangian formalism, CMES - Computer Modeling in Engineering and Sciences 14 (3) (2006) 181-198.

[45] Y. Vidal, J. Bonet, A. Huerta, Stabilized updated Lagrangian corrected SPH for explicit dynamic problems, Int. J. Numer. Methods Eng. 69 (13) (2007) $2687-2710$.

[46] P. W. Randles, L. D. Libersky, Normalized SPH with stress points, Int. J. Numer. Methods Eng. 48 (10) (2000) 1445-1462.

[47] S. Børve, M. Omang, J. Trulsen, Regularized smoothed particle hydrodynamics: A new approach to simulating magnetohydrodynamic shocks, Astrophys. J. 561 (1) (2001) 82-93.

[48] A. K. Chaniotis, D. Poulikakos, P. Koumoutsakos, Remeshed smoothed particle hydrodynamics for the simulation of viscous and heat conducting flows, J. Comput. Phys. 182 (1) (2002) 67-90. 
[49] A. K. Chaniotis, C. E. Frouzakis, J. C. Lee, A. G. Tomboulides, D. Poulikakos, K. Boulouchos, Remeshed smoothed particle hydrodynamics for the simulation of laminar chemically reactive flows, J. Comput. Phys. 191 (1) (2003) 1-17.

[50] S. E. Hieber, J. H. Walther, P. Koumoutsakos, Remeshed smoothed particle hydrodynamics simulation of the mechanical behavior of human organs, Technology and Health Care 12 (4) (2004) 305-314.

[51] J. Kuhnert, General smoothed particle hydrodynamics, Ph.D. thesis, Department of Mathematics, University of Kaiserslautern (1999).

[52] S. J. Cummins, M. Rudman, An SPH projection method, J. Comput. Phys. 152 (2) (1999) 584-607.

[53] J. Pozorski, A. Wawrenczuk, Sph computation of incompressible viscous flows, J. Theor. Appl. Mech. 40 (2002) 917-937.

[54] S. Shao, E. Y. M. Lo, Incompressible SPH method for simulating newtonian and non-newtonian flows with a free surface, Adv. Water Resour. 26 (7) (2003) $787-800$.

[55] F. Colin, R. Egli, F. Y. Lin, Computing a null divergence velocity field using smoothed particle hydrodynamics, J. Comput. Phys. 217 (2) (2006) 680-692.

[56] J. P. Hughes, D. I. Graham, Degradation and instability of incompressible SPH: Computations of simple viscous flows, in: A. J. C. Crespo (Ed.), SPHERIC Second International Workshop, Universidad Politcnica de Madrid, Madrid, Spain, 2007, pp. 83-86.

[57] X. Y. Hu, N. A. Adams, An incompressible multi-phase SPH method, J. Comput. Phys. 227 (1) (2007) 264-278.

[58] S. Koshizuka, H. Tamako, Y. Oka, A particle method for incompressible viscous flow with fluid fragmentation, Comput. Fluid Dyn. J. 4 (1) (1995) 29-46.

[59] S. Koshizuka, A. Nobe, Y. Oka, Numerical analysis of breaking waves using the moving particle semi-implicit method, Int. J. Numer. Methods Fluids 26 (7) (1998) 751-769.

[60] M. Ellero, M. Serrano, P. Español, Incompressible smoothed particle hydrodynamics, J. Comput. Phys. 226 (2) (2007) 1731-1752.

[61] A. Chorin, Numerical solution of the Navier-Stokes equations, J. Math. Comput. 22 (1968) 745-762.

[62] R. W. Hockney, J. W. Eastwood, Computer Simulation Using Particles, McGraw-Hill, New York, 1981.

[63] W. H. Press, S. A. Teukolsky, W. T. Vetterling, B. P. Flannery, Numerical Recipes in FORTRAN: The Art of Scientific Computing, 2nd Edition, Cambridge University Press, Cambridge, 1992.

[64] G. H. Cottet, P. D. Koumoutsakos, Vortex Methods: Theory and Practice, Cambridge University Press, London, 2000. 
[65] D. Xiu, S. J. Sherwin, S. Dong, G. E. Karniadakis, Strong and auxiliary forms of the semi-Lagrangian method for incompressible flows, J. Sci. Comput. 25 (1) (2005) 323-346.

[66] M. Falcone, R. Ferretti, Convergence analysis for a class of high-order semilagrangian advection schemes, SIAM J. Numer. Anal. 35 (3) (1998) 909-940.

[67] S. Tiwari, J. Kuhnert, Finite pointset method based on the projection method for simulations of the incompressible Navier-Stokes equations, in: M. Griebel, M. Schweitzer (Eds.), Meshfree Methods for Partial Differential Equations, Vol. 26 of Lecture Notes in Computational Science and Engineering, Springer, Berlin, 2003, pp. 373-388.

[68] E. Erturk, T. C. Corke, C. Gökçöl, Numerical solutions of 2-d steady incompressible driven cavity flow at high reynolds numbers, Int. J. Numer. Methods Fluids 48 (7) (2005) 747-774. 